\title{
Semiconductor Nanocrystals for Biological Imaging
}

\author{
Aihua $\mathrm{Fu}^{1^{*}}$, Weiwei $\mathrm{Gu}^{2 *}$, Carolyn Larabell ${ }^{2,3}$ and A. Paul Alivisatos ${ }^{1,4 \dagger}$ \\ ${ }^{1}$ Department of Chemistry, University of California, Berkeley, CA 94720, U.S.A. \\ ${ }^{2}$ Department of Anatomy, University of California, San Francisco, CA 94143, U.S.A. \\ ${ }^{3}$ Physical Bioscience Division, Lawrence Berkeley National Laboratory, Berkeley, CA \\ 94720, U.S.A. \\ ${ }^{4}$ Materials Science Division, Lawrence Berkeley National Laboratory, Berkeley, CA \\ 94720; U.S.A. \\ E-mails: aihuafu@uclink.berkeley.edu, wwgu@1bl.gov, larabell@lbl.gov, \\ alivis@uclink4.berkeley.edu
}

*These authors contributed equally to this work.

${ }^{\dagger}$ Corresponding Author: A. Paul Alivisatos, Department of Chemistry, University of California, Berkeley, CA 94720, Email: alivis@uclink4.berkeley.edu, Phone: 510-6437371, Fax: 510-642-6911

Key words: Semiconductor nanocrystals, quantum dots, fluorescent probe, imaging. 


\section{Summary of Recent Advances}

Conventional organic fluorophores suffer from poor photo stability, narrow absorption spectra and broad emission feature. Semiconductor nanocrystals, on the other hand, are highly photo-stable with broad absorption spectra and narrow size-tunable emission spectra. Recent advances in the synthesis of these materials have resulted in bright, sensitive, extremely photo-stable and biocompatible semiconductor fluorophores. Commercial availability facilitates their application in a variety of unprecedented biological experiments, including multiplexed cellular imaging, long-term in vitro and in vivo labeling, deep tissue structure mapping and single particle investigation of dynamic cellular processes. Semiconductor nanocrystals are one of the first examples of nanotechnology enabling a new class of biomedical applications.

\section{Introduction}

Semiconductor nanocrystals, also called quantum dots (QDs), are a new class of fluorescent biological labels. Originating from quantum confinement of electrons and holes within the nanocrystal core material, the fluorescence from QDs is unique compared to that from traditional organic fluorophores. For example, QDs exhibit high photo stability, broad absorption and narrow and symmetric emission spectra, slow excited state decay rate and large absorption cross section [1]. Their emission color can be continuously tuned from ultraviolet to visible and infrared wavelengths by changing the size and chemical composition of the semiconductor core nanocrystal. Growing a semiconductor shell with a larger band gap improves the quantum confinement resulting in very bright and highly stable, chemically as well as optically, semiconductor 
fluorophores [2,3]. QDs offer an exciting potential to overcome many of the limitations encountered by traditional organic dyes and genetically engineered fluorescent proteins. Since their introduction into biological imaging in 1998 [4,5], an enormous body of research has emerged focusing on the synthesis, photophysical property characterization and bioconjugation [6-9] of QDs. Advanced molecular and cellular imaging with QDs has also been realized $[10,11]$.

Biocompatible QDs find utility not only as a basic bio-labeling tool, but also as a key building block for complex multi-functional bio-probes. Their large surface area may be tailored to bind both target selective molecules and therapeutic molecules, enabling spontaneous delivery of treatments to a probed disease area. Complex nanostructures formed by linking QDs and gold nanoparticles through DNA hybridization or streptavidin-biotin interaction have also been realized [12-14] and applied in sensing biomolecular concentration [14]. Although QDs have been utilized in a broad range of imaging applications to date, their versatility for advanced biomedical applications remains to be fully explored. In this article, we will focus on applications in biological imaging, with a brief introduction about their unique optical properties, followed by a discussion of recent progresses in both in vitro and in vivo imaging as well as their application in neurobiology. Toxicity issues are also addressed.

\section{Optical Properties}

The size dependent optical properties of QDs result from their quantum-confined electronic states [15]. Just as in the "particle in a box" model, excitons in smaller nanocrystals experience stronger quantum confinement, resulting in larger photoluminescence energy. Figure 1 shows the typical absorption and emission spectra of 
water-soluble QDs. Their emission wavelength can be continuously tuned from $400 \mathrm{~nm}$ to $2000 \mathrm{~nm}$ by changing both the nanocrystal size and composition [16].

In contrast to conventional fluorophores, QDs have broad absorption and narrow and symmetric emission spectra. These features allow concurrent imaging of multiple entities in a single biological experiment, a quite difficult task with standard fluorophores since their relatively narrow excitation and broad emission spectra often result in spectra overlap [2]. Another advantage of QDs is that they are highly resistant to metabolic degradation and are hundreds of times more photo stable than conventional fluorophores. In addition, QDs often have a large Stokes shift, that is, a large separation between the excitation wavelength and the emission maxima, this has the effect of reducing autofluorescence, resulting in a several fold increase in sensitivity versus organic fluorophores [17].

For QDs, quantum yields can be as high as 0.89 at room temperature [18]; molar extinction coefficients, about $10^{5}-10^{6} \mathrm{M}^{-1} \mathrm{~cm}^{-1}$, are 10 to 100 times larger than most organic dyes [19]; and they have orders of magnitude larger two-photon absorption cross section [20]. Optical properties of QDs are usually unaffected by conjugation to biomolecules. Thus they are both highly stable and bright probes, especially suitable for photon-limited in vivo studies and continuous tracking experiments over extended time periods. A more extensive discussion of photophysical properties of QDs is presented by Grecco et al. [21]. 


\section{In Vitro Imaging}

QDs have been very successful in immunofluorescent labeling. With continuous efforts in developing high quality biocompatible QDs, nanoparticles conjugated to antibodies, peptides and DNA have been prepared and targeted to cells and tissues specifically, allowing multiplexed labeling and long term studies that can not be achieved by using standard dyes [22-29]. Although QDs and organic dyes can have comparable quantum yields, the larger absorption cross-section of the nanocrystal results in a much stronger photoluminescence signal. The sustained strong signal from a single nanoparticle was used to track dynamic cellular processes over time scales unavailable using organic fluorophores [10]. Recently, Dahan et al. developed a method to study single nanocrystal fluorescence patterns using defocused microscopy [30]. By relating these patterns to the structures of the nanocrystal emission dipoles they were able to determine the three-dimensional orientation of the nanoparticles, and successfully applied this technique to track the orientation of single membrane receptor in live cells (Figure 2). With continuous efforts in elucidating the photophysics of single QDs, there will be increasing interest in their application as fluorescent emitters for studying dynamic biophysical processes. For example, Yildiz and Selvin demonstrated that total internal reflection microscopy used in conjunction with organic fluorophores can produce fluorescence imaging with one nanometer accuracy (FIONA) [31]. In this technique, a large number of photons are collected over time from a single dye molecule, allowing researchers to locate the center of the fluorescent pattern with high precision. This technique has been applied to unravel the walking mechanism of the molecular motors myosin V, myosin VI and kinesin. Although the presented experimental results were 
from an organic dye, the authors believed that using QDs would provide at least a 10-fold improvement in time resolution and are extending the applications of FIONA in motor movements with QDs.

QDs can be uptaken by live cells with no need of any functionalization, possibly due to the characteristic size range and good biocompatibility. Pellegrino et al. studied the phagokinetic tracks left on a homogenous layer of silanized CdSe/ZnS, and demonstrated that QDs can be used as a two-dimensional in vitro invasion assay for discriminating between non-invasive and invasive cancer cell lines [32]. This technique provides a new tool for qualifying tumor cell invasiveness. Internalized QDs are also powerful probes for long-term studies of cell-cell interactions. They have been used to examine the interactions of human mammary epithelial tumor cells with normal cells growing in a 3D culture system. The tumor cell behavior around polarized normal cell clusters was clearly demonstrated when labeling tumor cells and normal cells with nanocrystals of different emission colors. The high photostability of the QDs is critical in the tracking and imaging of these cocultures for extended time periods (up to 14 days) and cannot be replaced by organic fluorophores (Figure 3, RM Boudreau, unpublished results).

Applying semiconductor nanoparticles for in vitro labeling allows fluorescent and electron microscopy $[10,18,33]$ imaging of the same probe, so that information on both temporal dynamics and high-resolution cellular localization can be obtained [10]. The fluorescence and electron density properties of QDs were also utilized by Nisman et al. to label a nuclear protein on cell sections and to correlate the fluorescence and TEM data. They also employed QDs in conjunction with immunogold to colocalize proteins at the ultrastructure level. Moreover, by obtaining cadmium elemental maps of $\mathrm{CdSe} / \mathrm{ZnS}$ 
distributed on a nuclear structure, the authors demonstrated the potential of using quantum dots as tags for electron spectroscopic imaging to colocalize multiple proteins [33].

\section{In vivo Imaging}

The extreme brightness of QDs and their resistance to photobleaching enable continuous exposure under laser illumination for an extended period of time, making them especially useful for in vivo imaging. Progress in nanocrystal synthesis, coating and surface modification has significantly enhanced their applications in tracking and imaging. Efforts in optimizing the surface coating for in vivo imaging have shown that specific polyethylene glycol (PEG) coatings result in longer circulation time [18,34], enhanced stability [18], and minimal nonspecific deposition [18,35], which are essential elements for in vivo imaging.

While the first in vivo targeting experiment imaged the histological sections of mouse organs after intravenous injection of peptide conjugated QDs, recent applications primarily focus on live animal imaging combined with multi-photon microscopy or with the use of near infrared nanocrystals.

The large two-photon absorption cross section of QDs allows for more efficient probing of thick specimens by multiphoton excitation microscopy [36]. With the use of this technique, fluorescence signals were able to be detected hundreds of microns deep through the skin of live mice [20] and thick tissue specimens [37]. Stroh and colleagues

recently explored the use of QDs in anatomical imaging with multiphoton microscopy. Unlike traditional fluorescence labeled dextran vessel markers, the nanocrystals distinctly 
differentiate tumor vessels from perivascular cells and matrix. This group also assessed the ability of nanocrystals to monitor tumor and cell trafficking [38]. These findings show the potential uses of QDs in designing drug delivery particles and tumor pathophysiological studies.

Tracking and imaging nanocrystals in live animals has been achieved by Nie's group. QDs were conjugated to the antibody specific for the prostate cancer cell marker PSMA. After injection into mice that had been transplanted with human prostate cancer cells, the quantum dot-tagged PSMA antibodies recognized and bound at the tumor site and were clearly imaged in vivo. Due to the large absorption coefficient and long lifetime, in vivo images of nanoparticles were much brighter and more sensitive than images with green fluorescent protein [34].

One challenge in live animal imaging is the significant autofluoresent background. Several strategies can be applied to solve this problem. One approach is to use spectral imaging or emission scanning microscopy to separate the nanocrystal fluorescence signal from background noise [34,37]. Since nanocrystals have narrow emission bands, this method also allows for multicolor tracking of up to five different nanoparticles in vivo [37]. An even more effective solution is to move from visible light to near infrared (NIR) since most tissue chromophores absorb weakly at such long wavelengths. Another advantage of NIR imaging is deeper penetration. Kim and coworkers first demonstrated the use of NIR QDs to map sentinel lymph nodes (SLN) during surgical procedures [11]. The nanoparticles, after intradermal injection into the animal, entered the lymphatic system, and were followed using an intraoperative imaging system. The surgeon followed the flow of nanocrystals in real time with NIR image 
guidance, and quickly identified the position of the SLN in a precise and rapid surgical procedure. NIR nanocrystal imaging of blood vessels and beating heart through 1-2 mm of skin and tissues were also reported [39].

\section{Semiconductor Nanocrystals for Neurobiology}

One common approach to studying neurotransporters involves the use of radiolabeled substrates or antagonists that can be monitored with high sensitivity. However, the cost and complexity involved in using radiolabeled material is high. Additionally, real time monitoring of the transporter activity is not possible. In contrast, a fluorescence based approaches allow for the localization and direct monitoring of realtime activities.

Owing to their high degree of photostability and brightness, QDs are more suitable probes than organic dyes for studies of neuronal protein or receptor dynamics over an extended period of time. Semiconductor nanoparticles have been used to track individual glycine receptors, a major inhibitory neurotransmitter receptor, on the surface

of cultured spinal neurons [10] (Figure 5). Compared to Cy3 dye, fluorescent nanoparticles had significant higher signal-to-noise ratio and allowed for tracking of single glycine receptors for at least 20 min, which is 200 times longer than Cy3 dye. Also, due to their small dimensions, nanoparticles are able to access dense synaptic regions and provide dynamical analysis that cannot be achieved with the use of $500 \mathrm{~nm}$ latex beads, one of the probes typically used for studying single molecule properties in live cells. 
Besides single molecule studies of neurotransmitters, QDs have been used to study neurotransmitter localization and signal pathways. Nanocrystals conjugated with peptides, antibodies, or other small molecules have been shown to recognize their target cell surface receptors [25,40-42]. It has also been shown that nanocrystal probes, after binding to their targets, can modulate receptor functions by either inhibiting ligand transportation [41] or activating downstream signaling [25,42].

Rosenthal and co-workers used nanocrystals conjugated with the neurotransmitter serotonin to target serotonin transporters on transfected cells [41]. Serotonin labeled nanocrystals specifically interacted with the serotonin receptor, and also inhibited the transportation of free serotonin in a way similar to antagonists.

Recently, Mason and coworkers studied norepinephrine (NE) and dopamine (DA) transporter (NET and DAT) locations and activities with semiconductor nanocrystal linked antibodies and peptides [40]. With streptavidin-biotin interaction, nanocrystals can specifically bind to NET in transfected cells as well as surface protein Limbic Associated Membrane Protein (LAMP) in hippocampal cultures. Since the activity of Ang II receptor is closely correlated with NET, the authors studied the localization of this receptor using nanocrystal-neuropeptide Ang II conjugates. This approach would allow for future study of Ang II receptor redistribution and dynamics in relation to NET activity in real-time.

To investigate whether QDs can serve as fluorescent nano-devices to evoke specific cell physiological responses, $\mathrm{Vu}$ et al. linked beta the subunit of neuron growth factor ( $\beta \mathrm{NGF})$ to the nanocrystal surface and used this complex to target tyrosine kinase A (TrkA) receptors of PC12 cells [42]. They reported that nanocrystal- $\beta$ NGF activated 
TrkA receptor initiated downstream signaling that resulted in conversion of PC12 cells to a neural phenotype.

These experiments show that QD-ligand conjugates are promising imaging probes for studying receptor-mediated activities and will have a wide range of applications in pharmaceutics and therapeutics.

\section{Toxicity}

Cadmium and selenium are known to be toxic [24]. Therefore, concerns have arisen about semiconductor nanocrystal toxicity and their environmental impact. Most of the above cell and animal experiments showed that when properly capped by both $\mathrm{ZnS}$ and hydrophilic shells, no obvious CdSe nanocrystal toxicity was observed under normal experimental conditions. Several groups have varied parameters such as synthesis, surface coating and incubation concentration to further investigate the potential toxicities of nanocrystals $[24,43-45]$. Cytotoxicity was observed when $\mathrm{Cd}^{2+}$ or $\mathrm{Se}^{2+}$ ions were released. This occurred when the nanoparticle surface coating was not stable, exposing the CdSe to oxidization by air or UV damage $[24,45]$. Surface molecules also play a role in QD cytotoxicity $[44,45]$. While cells can tolerate PEG-silica coated QDs at concentrations up to $30 \mu \mathrm{M}$, mercaptopropionic acid coated QDs have deleterious effect at $\sim 6 \mu \mathrm{M}[45]$.

Besides cytotoxicity, the degradation and metabolism of nanocrystals in the body remains to be investigated and there are reports that injected nanocrystals can accumulate in kidney, liver and spleen [34,35]. Whether nanocrystals can ultimately be cleared from 
the body is not known. More research in this area must be completed before they can be used as probes for diagnostic applications.

\section{Conclusions}

In the past several years, there has been increasing amount of interest in using QDs in an expanding variety of biological applications. Although they will not replace traditional fluorophores in biological imaging, QDs have been gradually accepted as a better alternative probes with enhanced signal-to-noise, extremely high stability, and improved specificity suitable for studying important biological problems.

\section{Acknowledgments}

The authors thank Shelley A. Claridge, Rosanne M. Boudreau, and Dr. Gerry McDermott for proofreading. This work was supported by NIH National Center for Research Resources through the Univ. of California, Los Angeles subaward agreement 0980GCD709 through the U.S. Dept. of Energy under Contract No. DE-AC0205CH11231, the DARPA/AFOSR DURINT Program Grant No. F49620-01-1-0474 under subcontract No. 066995 from the University of Southern California, and the Department of Defense Breast Cancer Research Program No. DAMD17-03-1-0637. 


\section{References:}

1. Michalet X, Pinaud F, Lacoste TD, Dahan M, Bruchez M, Alivisatos AP, Weiss S: Properties of Fluorescent Seminconductor Nanocrystals and their Applications to Biological Labeling. Single Mol. 2001, 2:261-276.

2. Dabbousi BO, Rodriguez-Viejo J, Mikulec FV, Heine JR, Mattoussi H, Ober R, Jensen KF, Bawendi M: (CdSe)ZnS core-shell quantum dots: synthesis and characterization of a size series of highly luminescent nanocrystallites. J phys Chem B. 1997, 101:94639475.

3. Hines MA, Guyot-Sionnest P: Synthesis and Characterization of Strongly

Luminescing ZnS-Capped CdSe Nanocrystals. J Phys Chem 1996, 100:468-471.

4. Bruchez M, Moronne M, Gin P, Weiss S, Alivisatos AP: Semiconductor

Nanocrystals as Fluorescent Biological Labels. Science 1998, 281:2013-2016.

5. Chan WC, Nie S: Quantum dot bioconjugates for ultrasensitive nonisotopic detection. Science 1998, 281:2016-2018.

6. Dubertret B, Skourides P, Norris DJ, Noireaux V, Brivanlou AH, Libchaber A: In vivo imaging of quantum dots encapsulated in phospholipid micelles. Science 2002, 298:1759-1762.

7. Han MY, Gao XH, Su JZ, Nie S: Quantum-dot-tagged microbeads for multiplexed optical coding of biomolecules. Nature Biotechnology 2001, 19:631-635.

8. Mattoussi H, Mauro JM, Goldman ER, Anderson GP, Sundar VC, Mikulec FV, Bawendi MG: Self-assembly of CdSe-ZnS quantum dot bioconjugates using an engineered recombinant protein. Journal of the American Chemical Society 2000, 122:12142-12150.

9. Qu LH, Peng XG: Control of photoluminescence properties of CdSe nanocrystals in growth. J Am Chem Soc 2002, 124:2049-2055.

*10. Dahan M, Levi S, Luccardini C, Rostaing P, Riveau B, Triller A: Diffusion dynamics of glycine receptors revealed by single-quantum dot tracking. Science 2003, 302:442-445. 
*11. Kim S, Lim YT, Soltesz EG, De Grand AM, Lee J, Nakayama A, Parker JA, Mihaljevic T, Laurence RG, Dor DM, et al.: Near-infrared fluorescent type II quantum dots for sentinel lymph node mapping. Nature Biotechnology 2004, 22:9397.

12. Fu AH, Micheel CM, Cha J, Chang H, Yang H, Alivisatos AP: Discrete nanostructures of quantum dots/Au with DNA. J Am Chem Soc 2004, 126:1083210833.

13. Gueroui Z, Libchaber A: Single-molecule measurements of gold-quenched quantum dots. Phys Rev Lett 2004, 93:166108.

14. Oh E, Hong MY, Lee D, Nam SH, Yoon HC, Kim HS: Inhibition assay of biomolecules based on fluorescence resonance energy transfer (FRET) between quantum dots and gold nanoparticles. $J$ Am Chem Soc 2005, 127:3270-3271.

15. Alivisatos AP: Perspectives on the physical chemistry of semiconductor nanocrystals. $J$ Phys Chem 1996, 100:13226-13239.

16. Gao X, Yang L, Petros JA, Marshall FF, Simons JW, Nie S: In vivo molecular and cellular imaging with quantum dots. Curr Opin Biotechnol 2005, 329:1173-1177.

17. Arya H, Kaul Z, Wadhwa R, Taira K, Hirano T, Kaul SC: Quantum dots in bioimaging: revolution by the small. Biochem Biophys Res Commun 2005, 329:1173-1177.

18. Ballou B, Lagerholm BC, Ernst LA, Bruchez MP, Waggoner AS: Noninvasive imaging of quantum dots in mice. Bioconjugate Chemistry 2004, 15:79-86.

19. Chan WC, Maxwell DJ, Gao X, Bailey RE, Han M, Nie S: Luminescent quantum dots for multiplexed biological detection and imaging. Curr Opin Biotechnol 2002, 13:40-46.

*20. Larson DR, Zipfel WR, Williams RM, Clark SW, Bruchez MP, Wise FW, Webb WW: Water-soluble quantum dots for multiphoton fluorescence imaging in vivo. Science 2003, 300:1434-1436.

21. Grecco HE, Lidke KA, Heintzmann R, Lidke DS, Spagnuolo C, Martinez OE, JaresErijman EA, Jovin TM: Ensemble and single particle photophysical proper-ties (TwoPhoton excitation, anisotropy, FRET, lifetime, spectral conversion) of commercial 
quantum dots in solution and in live cells. Microscopy Research and Technique 2004, 65:169-179.

22. Pinaud F, King D, Moore HP, Weiss S: Bioactivation and cell targeting of semiconductor $\mathrm{CdSe} / \mathrm{ZnS}$ nanocrystals with phytochelatin-related peptides. $J \mathrm{Am}$ Chem Soc 2004, 126:6115-6123.

**23. Michalet X, Pinaud FF, Bentolila LA, Tsay JM, Doose S, Li JJ, Sundaresan G, Wu AM, Gambhir SS, Weiss S: Quantum dots for live cells, in vivo imaging, and diagnostics. Science 2005, 307:538-544.

24. Derfus AM, Chan WCW, Bhatia SN: Probing the cytotoxicity of semiconductor quantum dots. Nano Letters 2004, 4:11-18.

25. Lidke DS, Nagy P, Heintzmann R, Arndt-Jovin DJ, Post JN, Grecco HE, JaresErijman EA, Jovin TM: Quantum dot ligands provide new insights into erbB/HER receptor-mediated signal transduction. Nature Biotechnology 2004, 22:198-203.

26. Kaul Z, Yaguchi T, Kaul SC, Hirano T, Wadhwa R, Taira K: Mortalin imaging in normal and cancer cells with quantum dot immuno-conjugates. Cell Res 2003, 13:503-507.

27. Xiao Y, Barker PE: Semiconductor nanocrystal probes for human metaphase chromosomes. Nucleic Acids Res 2004, 32:e28.

28. Gerion D, Chen FQ, Kannan B, Fu AH, Parak WJ, Chen DJ, Majumdar A, Alivisatos AP: Room-temperature single-nucleotide polymorphism and multiallele DNA detection using fluorescent nanocrystals and microarrays. Analytical Chemistry 2003, 75:4766-4772.

29. Chen F, Gerion D: Fluorescent CdSe/ZnS nanocrystal-peptide conjugates for long-term, nontoxic imaging and nuclear targeting in living cells. Nano Letters 2004, 4:1827-1832.

30. Brokmann X, Ehrensperger MV, Hermier JP, Triller A, Dahan M: Orientational imaging and tracking of single CdSe nanocrystals by defocused microscopy. Chemical Physics Letters 2005, 406:210-214. 
31. Yildiz A, Selvin PR: Fluorescence imaging with one nanometer accuracy: application to molecular motors. accounts of Chemical Research 2005, appears on Web.

32. Pellegrino T, Parak WJ, Boudreau R, Le Gros MA, Gerion D, Alivisatos AP, Larabell CA: Quantum dot-based cell motility assay. Differentiation 2003, 71:542-548.

33. Nisman R, Dellaire G, Ren Y, Li R, Bazett-Jones DP: Application of quantum dots as probes for correlative fluorescence, conventional, and energy-filtered transmission electron microscopy. Journal of Histochemistry \& Cytochemistry 2004, 52:13-18.

*34. Gao X, Cui Y, Levenson RM, Chung LWK, Nie S: In vivo cancer targeting and imaging with semiconductor quantum dots. Nat Biotechnol 2004, 22:969-976.

35. Akerman ME, Chan WC, Laakkonen P, Bhatia SN, Ruoslahti E: Nanocrystal targeting in vivo. Proc Natl Acad Sci U S A 2002, 99:12617-12621.

**36. Alivisatos AP, Gu W, Larabell C: Quantum Dots as Cellular Probes. Annual Review of Biomedical Engineering 2005, 7.

37. Voura EB, Jaiswal JK, Mattoussi H, Simon SM: Tracking metastatic tumor cell extravasation with quantum dot nanocrystals and fluorescence emission-scanning microscopy. Nature Medicine 2004, 10:993-998.

38. Stroh M, Zimmer JP, Duda DG, Levchenko TS, Cohen KS, Brown EB, Scadden DT, Torchilin VP, Bawendi MG, Fukumura D, et al.: Quantum dots spectrally distinguish multiple species within the tumor milieu in vivo. Nat Med 2005.

39. Morgan NY, English S, Chen W, Chernomordik V, Russo A, Smith PD, Gandjbakhche A: Real time in vivo non-invasive optical imaging using near-infrared fluorescent quantum dots. Acad Radiol 2005, 12:313-323.

40. Mason JN, Farmer H, Tomlinson ID, Schwartz JW, Savchenko V, DeFelice LJ, Rosenthal SJ, Blakely RD: Novel fluorescence-based approaches for the study of biogenic amine transporter localization, activity, and regulation. $J$ Neurosci Methods 2005, 143:3-25. 
41. Rosenthal SJ, Tomlinson A, Adkins EM, Schroeter S, Adams S, Swafford L, McBride J, Wang YQ, DeFelice LJ, Blakely RD: Targeting cell surface receptors with ligand-conjugated nanocrystals. Journal of the American Chemical Society 2002, 124:4586-4594.

42. Vu TQ, Maddipati R, Blute TA, Nehilla BJ, Nusblat L, Desai TA: Peptideconjugated quantum dots activate neuronal receptors and initiate downstream signaling of neurite growth. Nano Letters 2005, 5:603-607.

43. Hoshino a, Fujioka K, Oku T, Suga M, Sasaki YF, Ohta T, Yasuhara M, Suzuki K, Yamamoto K: Physicochemical properties and cellular toxicity of nanocrystal quantum dots depend on their surface modification. Nano Letters 2004, 4:2163-2169.

44. Shiohara A, Hoshino A, Hanaki K, Suzuki K, Yamamoto K: On the cyto-toxicity caused by quantum dots. Microbiol Immunol 2004, 48:669-675.

45. Kirchner C, Liedl T, Kudera S, Pellegrino T, Javier AM, Gaub HE, Stolzle S, Fertig N, Parak WJ: Cytotoxicity of colloidal CdSe and CdSe/ZnS nanoparticles. Nano Letters 2005, 5:331-338. 


\section{Figure Legend}

Figure 1. (a) Excitation (dashed) and fluorescence (solid) spectra of fluorescein (A) and a typical water-soluble QD sample (B) in PBS. The nanocrystals have a much narrower emission (32 $\mathrm{nm}$ compared with $45 \mathrm{~nm}$ at half maximum and $67 \mathrm{~nm}$ compared with $100 \mathrm{~nm}$ at $10 \%$ maximum), no red tail, and a broad, continuous excitation spectrum. (b) A, Size- and material-dependent emission spectra of several surfactant-coated QDs in a variety of sizes. The blue series represents different sizes of CdSe QDs with diameters of 2.1, 2.4, 3.1, 3.6, and $4.6 \mathrm{~nm}$ (from right to left). The green series is of InP QDs with diameters of 3.0, 3.5, and $4.6 \mathrm{~nm}$. The red series is of InAs QDs with diameters of 2.8, 3.6, 4.6, and $6.0 \mathrm{~nm}$. B, A true-color image of a series of silica-coated core (CdSe)shell ( $\mathrm{ZnS}$ or $\mathrm{CdS})$ nanocrystal probes in aqueous buffer all illuminated simultaneously with a handheld ultraviolet lamp. Reproduced with permission from [4].

Figure 2. Defocused microscopy images of QD coupled glycine receptor in the membrane of a Hele cell. The contour intensities (dotted lines) can be fitted (solid lines) to determine the orientation $(\Theta, \Phi)$ of each QD. Reproduced with permission from [30].

Figure 3. QDs were used to study mixed cell interactions in a 3-D Matrigel culture system. (A) Human mammalian epithelial MCF 10A cells (labeled with green emitting silica coated QDs) form acini structures after growing in growth-factor reduced matrigel for 10 days. (B) After the acini were formed, human breast tumor MDA-MB231 cells (labeled with red-emitting silica coated QDs) were added to the culture. After 
14-16 $\mathrm{h}$ of incubation, the tumor cells had attached to the acini. (C) The contact was fatal to the tumor cells, which were found dead surrounding the MCF 10A acinus. Most of the tumor cells had lysed, leaving transparent ghosts loosely attached to the acinus, but a few newly attached cells still retained red-emitting QDs. (D) The MCF-10A acini and all invading tumor cells; it is a superimposition of all sections, displaying the sharp edge of each cell followed by a projection of color-coded depth information so that red is the uninvolved lower portion of the MCF-10A acini and the tumor cells are shades of orange through green. Bar $=10 \mu \mathrm{m}$. From manuscript in preparation.

Figure 4. Near infrared (NIR) QD sentinel lymph node mapping in the mouse. The mouse was injected intradermally with 10pmol of NIR QDs in the left paw. Left, preinjection NIR autofluorescence image; middle, 5 min post injection white light color video images; right, 5 min post-injection NIR fluorescence image. An arrow indicates the putative axillary sentinel lymph node. Reproduced with permission from [11].

Figure 5. QDs as marker for glycine receptor (GlyR) localization in neurons. QD labeled GlyR (red) was detected over the somatodendritic compartment identified by microtubule-associated protein-2 (green). Arrows mark clusters of QD labeled GlyRs located on dendrites. Reproduced with permission from [10]. 


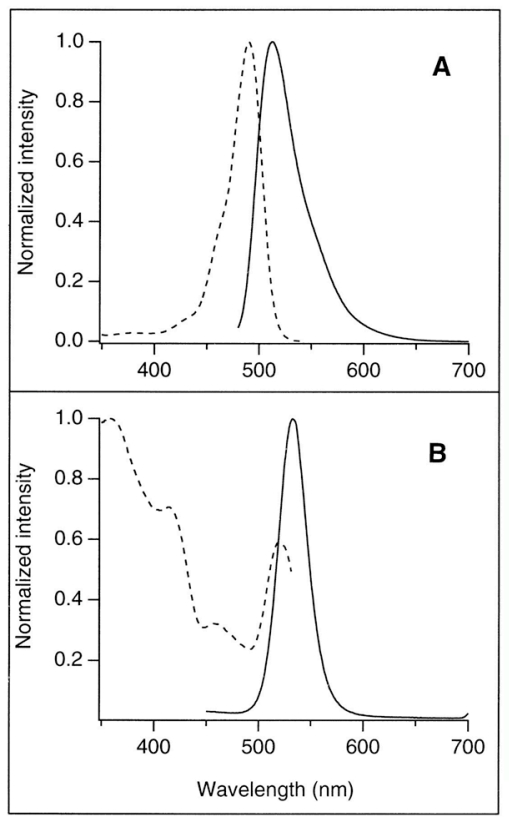

(a)

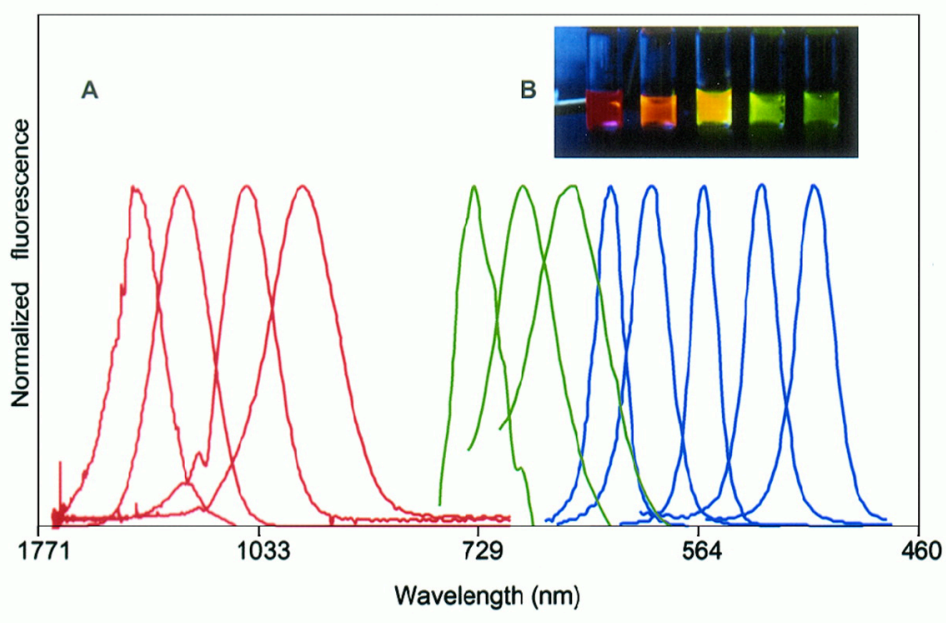

(b)

Figure 1 

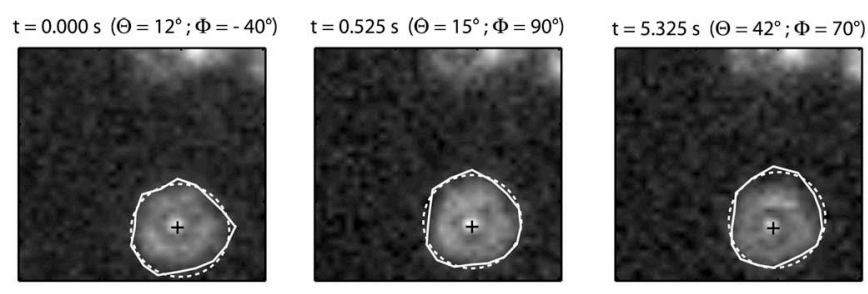

$\mathrm{t}=13.275 \mathrm{~s}\left(\Theta=33^{\circ} ; \Phi=70^{\circ}\right)$
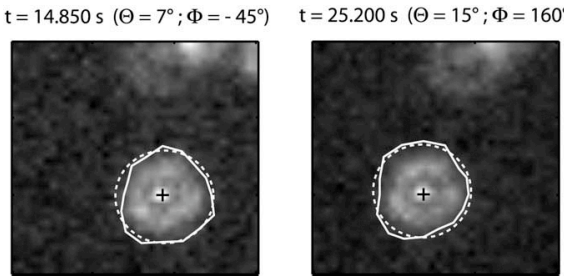

Figure 2 


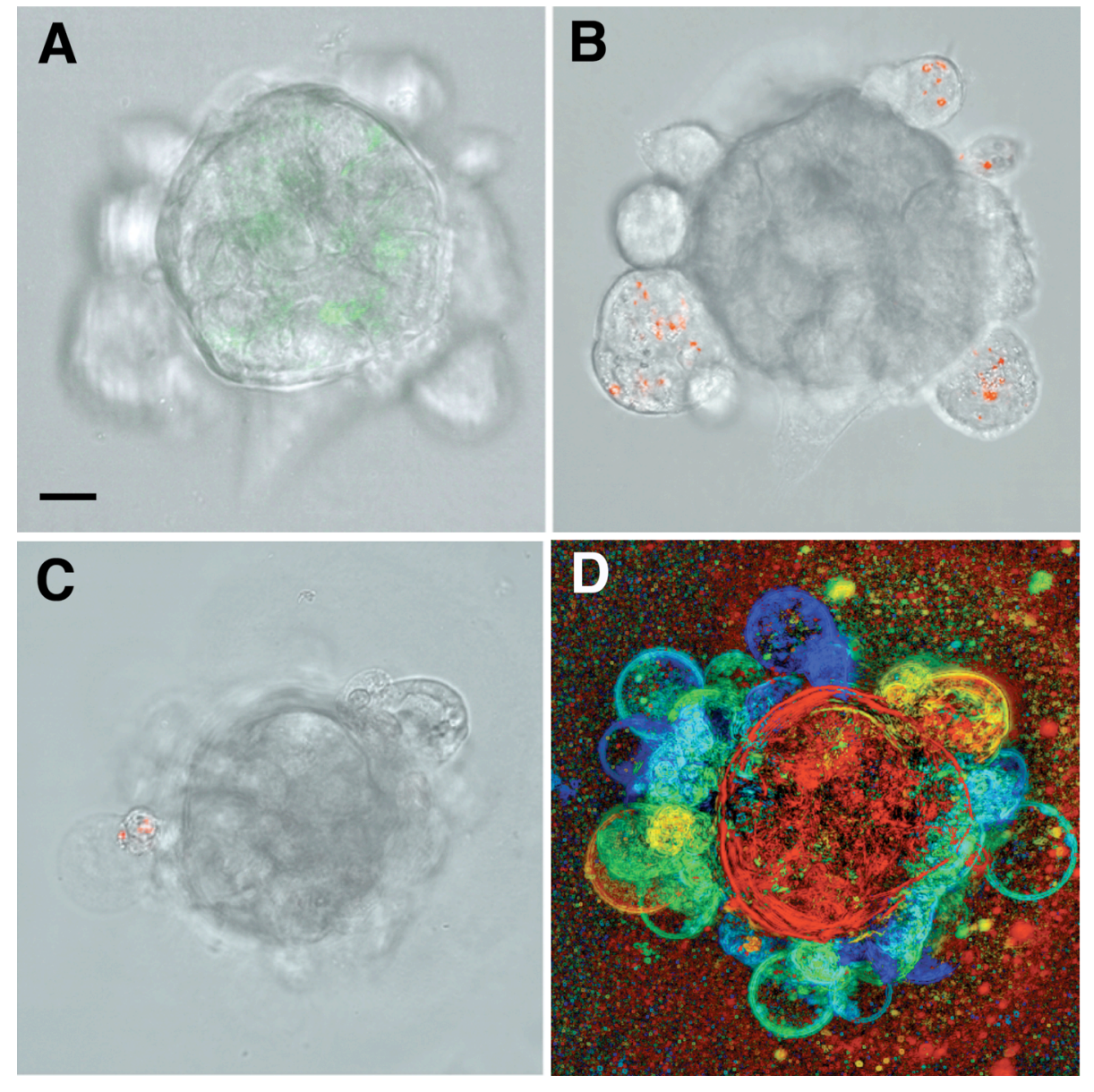

Figure 3 

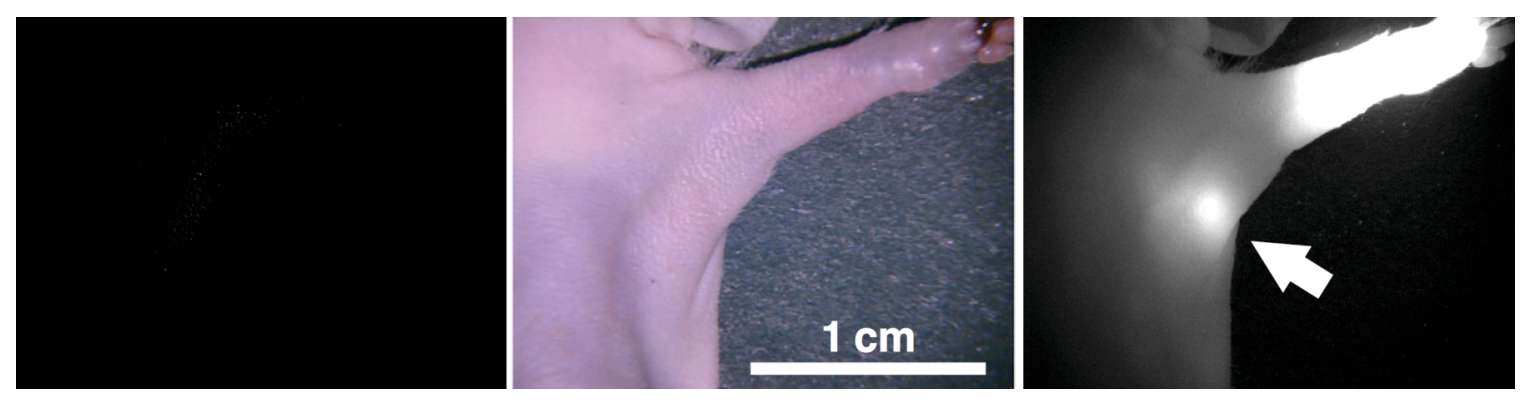

Figure 4 


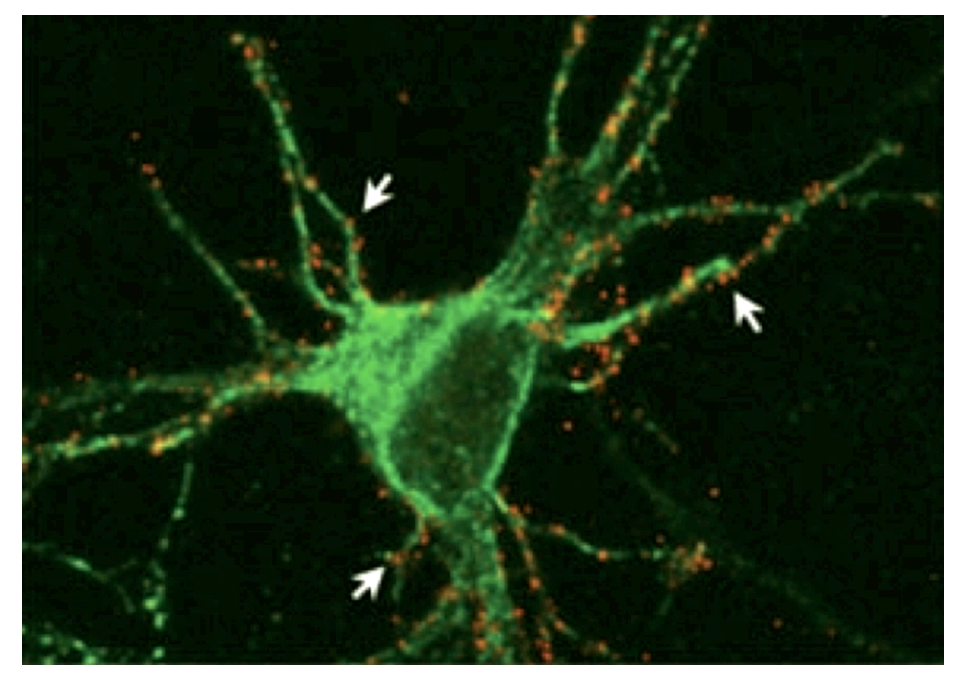

Figure 5

- 24 - 